\title{
Correlativity between osteocalcin and coronary artery calcification in middle-aged and elderly population
}

Na Li (D 18865381906@163.com)

Shandong University Qilu Hospital https://orcid.org/0000-0002-1538-1243

Zhimian Zhang

Shandong University Qilu Hospital

Research article

Keywords: vascular calcification, Coronary artery calcification, osteocalcin, N- MID

Posted Date: June 5th, 2020

DOI: https://doi.org/10.21203/rs.3.rs-29979/v1

License: (c) (1) This work is licensed under a Creative Commons Attribution 4.0 International License.

Read Full License 
Article title: Correlativity between osteocalcin and coronary artery calcification in middle-aged and elderly population

Author names: $\mathrm{Na} \mathrm{Li}^{1}$, Zhi-Mian Zhang ${ }^{1}$

Corresponding author: Zhi-Mian Zhang

Author affiliation: ${ }^{1}$ Department of Health Management Center, Qilu Hospital, Shandong University, Jinan, China

Mailing address : Zhi-Mian Zhang, Department of Health Management Center,Qilu Hospital, Shandong University, No. 107, West Wenhua Road, Jinan, Shandong 250012, China. Email: zhangzhimian@126.com

Abstract: Background and Objective: With the increase of aging, osteoporosis and cardiovascular diseases are common diseases in middle-aged and elderly people. The purpose of this study is to explore the correlation between osteocalcin and coronary calcification.

Methods: A total of 150 middle-aged and elderly physical examination subjects who had undergone coronary artery CT angiography (CTA) were selected as the research objects. The calcification score of the coronary artery was calculated according to the Agatston (AS) integral method, and the total score was divided into the coronary artery calcification group and the non-calcification group. The indexes of $\mathrm{N}$-terminal midfragment of osteocalcin (N-MID), routine biochemical indexes and basic conditions of each patient were recorded.

Results: In the comparison of general data between the non-calcification group and the calcification group, the differences in age $(\mathrm{P}<0.001)$, pressure difference $(\mathrm{P}=0.01)$ and low density lipoprotein cholesterol (LDL-C) $(\mathrm{P}<0.001)$ were statistically significant, the differences in smoking history $(\mathrm{P}<0.001)$ and drinking history $(\mathrm{P}<$ $0.001)$ were statistically significant, and the differences in N-MID $(\mathrm{P}<0.001)$ were statistically significant. Coronary artery calcification score (CACS) was correlated with age, pressure difference, LDL-C, N-MID, smoking history and drinking history. The four variables, age, LDL, N-MID, and smoking history, have certain effects on coronary artery calcification (CAC).

Conclusions: In middle-aged and elderly people undergoing physical examination, N- 
MID is correlated with CAC and can be used as a predictive indicator.

\section{Abbreviations :}

AS: Agatston

BGLAP: Bone glutamate protein

BMI: Body mass index

CABG: Coronary artery bypass grafting

CAC: Coronary calcification calcification

CACS: Coronary artery calcification score

CAD: Coronary artery disease

CHD: Coronary heart disease

CKD: Chronic renal failure

cOC: Carboxylated osteocalcin

CTA: Coronary artery CT angiography

CVD: Cardiovascular disease

FBG: Fasting blood glucose

GGCX: Glutamate $\gamma$-carboxylase

HDL-C: High density lipoprotein cholesterol

HR: Heart rate

LDL-C: Low density lipoprotein cholesterol

N-MID: N-terminal midfragment of osteocalcin

OC: Osteocalcin

TC: Total cholesterol

TG: Triglyceride

uOC: Uncarboxylated osteocalcin

VC: Vascular calcification

VKDPs: Vitamin k-dependent proteins

VSMCs: Vascular smooth muscle cells

$\bar{x} \pm s:$ Mean \pm standard deviation

Keywords: vascular calcification, Coronary artery calcification, osteocalcin, N- MID 


\section{Background}

By the end of 2017, there were 2.409 million elderly people over the age of 60 in the country ${ }^{1}$. With the increase of aging, the health problems of the elderly become more and more prominent. Osteoporosis and cardiovascular disease (CVD) are the two most common diseases among the elderly, and some studies have suggested that there may be a link between the wo $^{2-5}$. As people get older, the calcium lost from bones is deposited in the cardiovascular system ${ }^{6}$. Vascular calcification (VC) is a recognized risk factor for cardiovascular morbidity and mortality ${ }^{7}$ and is currently considered an active process $^{8-11}$.VC has some similarities to bone mineralization and is related to the transformation of vascular smooth muscle cells (VSMCs) into osteoblast-like phenotypes $^{12}$.

There are 17 types of vitamin k-dependent proteins (VKDPs), including osteocalcin (OC) [also known as bone glutamate protein (BGLAP)]. OC is divided into two kinds: carboxylated osteocalcin (cOC) and uncarboxylated osteocalcin (uOC). uOC is converted into cOC under the action of glutamate $\gamma$-carboxylase (GGCX) and vitamin $\mathrm{K}^{13}$. OC is the most abundant non-collagenous protein in the bone mineralization matrix $^{14}$. The characteristic of atherosclerosis is associated with circulating $\mathrm{OC}^{15}$. OC mediates calcification of $\mathrm{VSMCs}^{16}$. These studies show that OC is correlated with VC.

At present, the relationship between $\mathrm{OC}$ and $\mathrm{VC}$ has not been determined. Research on cOC is still lacking. In this paper, one of the indexes of bone metabolism - N-terminal midfragment of osteocalcin (N-MID) was included to investigate its relationship with coronary artery calcification (CAC).

\section{Methods}

\subsection{Study population}

A total of 150 middle-aged and elderly physical examination subjects who had undergone coronary artery CT angiography (CTA) were selected as the research objects. The exclusion criteria for all selected candidates are: diseases that affect bone metabolism, such as hyperthyroidism, hyperparathyroidism, chronic renal failure or malignancy; or use drugs that affect bone metabolism, such as glucocorticoids, 
estrogens, or bisphosphonates; patients with incomplete clinical data; previous history of fracture; acute heart, liver, kidney, lung and other important organ complications occurred within the last 3 months. All participants in this study agreed in writing.

\subsection{General data}

Retrospective data analysis was used to record the subjects' gender、age、 body mass index (BMI)、systolic blood pressure、 diastolic blood pressure、 differential blood pressure 、 heart rate $(\mathrm{HR})$ 、 fasting blood glucose $(\mathrm{FBG})$ 、 triglyceride $(\mathrm{TG})$, total cholesterol (TC)、 low density lipoprotein cholesterol (LDL-C)、 high density lipoprotein cholesterol (HDL-C)、 smoking history 、 drinking history and medication history. Measurement of blood pressure: after resting for at least 10 minutes, take an average of two readings of blood pressure under the sitting position. Measurement of routine biochemical indicators: After one night of fasting, each participant collected blood samples of forearm veins in the morning and sent them to the laboratory of our hospital to complete relevant index tests.

\subsection{Measurement of N-MID}

The complete osteocalcin (amino acid 1-49) is not very stable in the peripheral blood. The amino acid chain between the carboxyl terminus 43 and 44 is easily hydrolyzed by protease. The large fragment (amino acid 1-43) after cleavage is called N-MID. N-MID is relatively stable and is an internationally recognized marker of bone metabolism. The detection of N-MID can better reflect the recent bone transformation. We used the third generation N-MID assay kit to detect the whole 1-49 of cOC and 143 of degraded cOC fragments by enzyme-linked immunoassay.

\subsection{Measurement of CAC score}

Multi-slice spiral CT was used to detect the calcification score of coronary arteries ${ }^{17}$, The calcification integral was calculated by the Agatston (AS) integral method, AS calcification integral condition: CT value $\geq 130 \mathrm{HU}$, calcification area $\geq 1 \mathrm{~mm}^{2}$, calcification score $=$ calcification area $\times$ calcification peak $\operatorname{score}(130 \sim 199 \mathrm{Hu}=1,200 \sim 299 \mathrm{Hu}=2,300 \sim 399 \mathrm{Hu}=3, \geq 400 \mathrm{Hu}=4)$. The coronary artery was divided into four parts: left main trunk, left anterior descending branch, left circumflex branch, and right coronary artery, and calcification scores were calculated 
for each part. The integral results of each part are added to obtain a total integral ${ }^{18}$. According to the total score, this study was divided into the coronary artery calcification group and the non-calcification group (calcification group: $>10$; non-calcification group: $\leq 10)$.

\subsection{Statistical analysis}

Statistical software SPSS19.0 was used for statistical analysis. The measurement data is expressed in mean \pm standard deviation $(\bar{x} \pm s)$, the counting data is represented by probability, and the grade data by frequency. For comparison of measurement data, the independent data $t$ test is used if the data conforms to normal distribution and uniform variance, and the Wilcoxon rank sum test is used if data does not conform to normal distribution or uneven variance. The comparison of counting data was conducted by $\chi^{2}$ test, and Spearman rank correlation analysis was used for correlation analysis. Binary Logistic regression was used to analyze the correlation between multiple variables and coronary artery calcification score. The test level was $\alpha=0.05$, and $P<0.05$ was considered statistically significant.

\section{Results}

3.1 Baseline characteristics of the study population are shown in Table 1. Comparison of the general data between the two groups showed statistically significant differences in age $(P<0.001)$, pressure difference $(P=0.01)$ and LDL-C $(P<0.001)$, and differences in smoking history $(P<0.001)$ and drinking history $(P<0.001)$ were statistically significant. There was no statistically significant difference in gender, height, weight, BMI, systolic blood pressure, diastolic blood pressure, HR, FBG, TG, TC and HDL-C $(P>0.05)$.

Tab.1 Comparison of baseline characteristics of 150 subjects. Data are given as n (\%) or $\bar{x} \pm s$.

\begin{tabular}{llll}
\hline & $\begin{array}{l}\text { Non-calcification group } \\
(n=72)\end{array}$ & $\begin{array}{l}\text { Calcification group } \\
(n=78)\end{array}$ & $P$ Value \\
\hline Gender (male/female) & $31 / 41(47.00 / 48.80)$ & $41 / 28(53.00 / 51.20)$ & 0.82 \\
Age (years) & $54.17 \pm 6.97$ & $60.19 \pm 9.21$ & $<0.001$ \\
Height $(\mathrm{cm})$ & $164.40 \pm 7.85$ & $164.62 \pm 7.09$ & 0.86
\end{tabular}




\begin{tabular}{llll} 
Weight $(\mathrm{kg})$ & $72.33 \pm 12.71$ & $69.49 \pm 12.14$ & 0.16 \\
BMI $\left(\mathrm{kg} / \mathrm{m}^{2}\right)$ & $26.67 \pm 3.74$ & $25.54 \pm 3.59$ & 0.06 \\
Systolic blood pressure $(\mathrm{mmHg})$ & $136.74 \pm 20.32$ & $140.79 \pm 18.62$ & 0.20 \\
Diastolic blood pressure $(\mathrm{mmHg})$ & $81.64 \pm 12.15$ & $79.08 \pm 12.22$ & 0.20 \\
Pressure difference(mmHg) & $55.10 \pm 13.38$ & $61.72 \pm 17.09$ & 0.01 \\
HR(beats/min) & $73.88 \pm 10.56$ & $74.31 \pm 12.20$ & 0.82 \\
FBG $(\mathrm{mmol} / \mathrm{L})$ & $5.86 \pm 1.34$ & $6.14 \pm 1.93$ & 0.30 \\
TG $(\mathrm{mmol} / \mathrm{L})$ & $1.61 \pm 0.73$ & $1.61 \pm 0.85$ & 0.99 \\
TC $(\mathrm{mmol} / \mathrm{L})$ & $4.64 \pm 1.01$ & $4.74 \pm 1.07$ & 0.54 \\
HDL-C(mmol/L) & $1.28 \pm 0.28$ & $1.23 \pm 0.24$ & 0.22 \\
LDL-C(mmol/L) & $2.52 \pm 0.71$ & $3.14 \pm 0.74$ & $<0.001$ \\
Smoking history & $12(1.40)$ & $38(27.50)$ & $<0.001$ \\
Drinking history & $6(19.40)$ & $25(80.60)$ & $<0.001$ \\
\hline
\end{tabular}

$\mathrm{BMI}=$ Weight $/ \mathrm{height}^{2}\left(\mathrm{~kg} / \mathrm{m}^{2}\right)$; Pressure difference $=$ Systolic blood pressure- Diastolic blood pressure $(\mathrm{mmHg})$.

3.2 The relationship between CAC and N-MID is shown in table 2. The relationship between CAC and N-MID was statistically significant when comparing without gender distinction $(P<0.001)$. When comparing the relationship between CAC and N-MID in male $(P=0.005)$ and female $(P<0.001)$, the difference was still statistically significant.

Tab.2 The relationship between N-MID and CAC was discussed by gender stratification.

\begin{tabular}{lccc}
\hline & $\begin{array}{c}\text { Non-calcification group } \\
(n=72)\end{array}$ & $\begin{array}{c}\text { Calcification group } \\
(n=78)\end{array}$ & $P$ Value \\
\hline Without gender distinction & $10.49 \pm 5.47$ & $16.24 \pm 5.87$ & $<0.001$ \\
Male & $10.72 \pm 6.57$ & $14.66 \pm 4.19$ & 0.005 \\
Female & $10.31 \pm 4.55$ & $17.52 \pm 6.73$ & $<0.001$ \\
\hline
\end{tabular}

3.3 Correlation analysis of CAC with N-MID and general data in table 3. Correlation analysis was performed by Spearman rank correlation analysis. The results showed that the difference between CAC and age $(r=0.35, P<0.001)$, pressure difference $(r=0.21$, $P=0.01), \operatorname{LDL}(r=0.39, P<0.001), \mathrm{N}-\mathrm{MID}(r=0.45, P<0.001)$, smoking history $(r=0.29$, 
$P<0.001)$ and drinking history $(r=0.34, P<0.001)$ at the $\mathrm{P}=0.05$ level was statistically significant. Binary Logistic regression was used to evaluate the influence of multiple variables on $\mathrm{CAC}$, and the difference of Logistic model was statistically $\operatorname{significant}\left(\chi^{2}\right.$ $=82.098, P<0.001$ ). Among the independent variables (or predictors) included in this model, the four variables of age $(P=0.02), \operatorname{LDL}(P=0.002), \mathrm{N}-\operatorname{MID}(P<0.001)$ and smoking history $(P=0.02)$ had statistically significant differences at the level of $\mathrm{P}=0.05$.

Tab.3 Results of Logistic model analysis

\begin{tabular}{llll}
\hline & OR & $95 \% C I$ & $P$ Value \\
\hline Age & 1.08 & $(1.02 \sim 1.15)$ & 0.02 \\
Pressure difference & 1.02 & $(0.99 \sim 1.05)$ & 0.27 \\
LDL & 2.71 & $(1.45 \sim 5.10)$ & 0.002 \\
N- MID & 1.23 & $(1.11 \sim 1.37)$ & $<0.001$ \\
Smoking history & 4.38 & $(1.33 \sim 14.45)$ & 0.02 \\
Drinking history & 2.20 & $(0.81 \sim 5.97)$ & 0.12 \\
\hline
\end{tabular}

\section{Discussion}

VC is very common in patients with ischemic cardiovascular disease, cerebrovascular disease and renal failure. Vascular calcification is a major factor in the progression of cardiovascular disease ${ }^{19}$. Calcified vascular cells are derived from local VSMCs and circulating hematopoietic stem cells (especially intimal calcification) ${ }^{20}$. Vascular calcification is a marker of atherosclerosis ${ }^{21}$. In calcified atherosclerotic lesions, VSMCs express a variety of osteogenic differentiation markers ${ }^{22}$, similar to the osteogenic mineralization process ${ }^{23}$. VC is caused by the imbalance of vascular wall mineralization promoters ${ }^{24}$ and inhibitors and is a serious health problem with serious clinical consequences ${ }^{25}$. CAC is an independent predictor of coronary heart disease(CHD) / $\mathrm{CVD}^{26}$. Compared with other risk markers, it has better identification and risk reclassification ability ${ }^{27-29}$, which is related to the degree of coronary artery disease $(C A D)^{30}$. Studies have shown that in the early stage of dialysis patients with chronic renal failure(CKD), CAC is closely related to the incidence of CVD events and 
all-cause mortality ${ }^{31}$.

There are many risk factors for CVD, including age, diabetes, hypertension, hyperlipidemia and so on. The results of this study showed that age, LDL and other general data were significantly higher in the calcified group than in the non-calcified group. In this study, differential pressure was added, and the results showed that the differential pressure was significantly different between the two groups and correlated with the CACS.

Some studies have found that the progressive calcification of atherosclerotic plaque is accompanied by the accumulation of insignificant calcitonin and osteocalcin, and the concentration of $\mathrm{OC}$ is significantly increased during the calcification process ${ }^{32}$. A meta-analysis ${ }^{33}$ evaluated the relationship between circulating OC(total OC, uOC, and cOC) and cardiovascular outcomes. Patients with CVD had significantly lower circulating total OC levels than patients with non- CVD. Data on $\mathrm{uOC}$ and cOC were limited and there was no evidence of association. Studies have evaluated the OC levels of subjects undergoing coronary artery bypass grafting (CABG) compared with the normal control group. Regardless of diabetes status, serum uOC levels have decreased, and there is no difference in cOC levels between the two groups ${ }^{34}$. A prospective cohort study examining the association of various bone markers with CVD risk in type 2 diabetes found no association between OC and CVD risk ${ }^{35}$. Studies have found that there is no difference in serum OC levels between stroke patients and healthy controls ${ }^{36}$. A meta-analysis found no clear association between $\mathrm{OC}$ and $\mathrm{VC}$ or the degree of atherosclerosis $^{14}$.

OC is a non-specific collagen produced by osteoblasts in the bone and consists of 49 amino acids. Previous studies have not come to a uniform conclusion. This study included the N-MID indicator, we used the third generation n-mid assay kit to detect the whole 1-49 of cOC and 1-43 of degraded cOC fragments by enzyme-linked immunoassay. The metabolism of bone tissue can be understood by detecting the level of n-mid in blood. In this paper, the relationship between N-MID and CAC was compared. We found that compared with the non-calcification group, the value of $\mathrm{N}$ MID in the calcification group was higher, and the difference was statistically 
significant. This article also proves that N-MID is related to CAC and has a certain impact on CAC. This paper also discusses the relationship between n-mid and CAC through gender stratification, and finds that there is still a correlation between the two, whether male or female. Future research may focus on the relationship between different types of OC and VC to draw relevant conclusions.

Why come to a different conclusion? The forms of osteocalcin used in each study were different, including total $\mathrm{OC}, \mathrm{cOC}$ and $\mathrm{Uoc}$; the reagents used to detect $\mathrm{OC}$ varies; different gender selection; study population diversity (kidney disease, diabetes or glucose intolerance, postmenopausal women, and hypertension); ethnic and regional variability; blood sampling time varies; The calcification or atherosclerosis measurement methods used vary, including calcification scoring, intima-media thickness measurement, pulse wave velocity measurement, plaque presence, and coronary angiography or echocardiography; Comorbidities increase with age, which may influence the findings of some studies. In addition, OC concentrations were also affected by drug treatments, including glucocorticoid therapy, antiabsorbents, and vitamin D therapy. Not all researchers believe that these are potential confounders.

\section{Conclusion}

In middle-aged and elderly people undergoing physical examination, N-MID is correlated with $\mathrm{CAC}$ and can be used as a predictive indicator.

\section{Declarations:}

Ethics approval and consent to participate: All procedures performed in studies involving human participants were in accordance with the ethical standards of the institutional and/or national research committee and with the Declaration of Helsinki and have been approved by Ethics committee of Shandong university Qilu hospital. Written informed consent was obtained from individual or guardian participants.

Consent for publication: Not applicable.

Availability of data and materials: All data generated or analyzed during this study are included in this published article.

Competing interests: The authors declare that they have no competing interests. 
Funding: Major science and technology innovation project of Shandong province (2019JZZY011110). To pay for the experiment and the manuscript.

Authors' contributions: $\mathrm{Na}$ Li collected a variety of clinical information and analyzed it to reach conclusions. $\mathrm{Na} \mathrm{Li} \mathrm{was} \mathrm{a} \mathrm{major} \mathrm{contributor} \mathrm{in} \mathrm{writing} \mathrm{the} \mathrm{manuscript.} \mathrm{Zhi} \mathrm{-}$ Mian Zhang provided this topic and conducted a clinical study. All authors read and approved the final manuscript.

Acknowledgements: We appreciated professor Gao for providing the testing reagent.

\section{References}

${ }^{1}$ Wang HM. Attaching importance to health of elderly population and promoting national healthy ageing actively in China[J]. Zhonghua Liu Xing Bing Xue Za Zhi, 2019,40(3):259-265.

2 Zhou R, Zhou H, Cui M, et al. Association Between Aortic Calcification and the Risk of Osteoporosis in a Chinese Cohort: The Chongqing Osteoporosis Study[J]. Calcif Tissue Int, 2013,93(5):419-25.

3 Verheyen N, Grübler MR, Catena C, et al. The bone-cardiovascular axis: mechanisms and clinical relevance[J]. Int J Endocrinol, 2018, 2018.

4 Laroche M, Pécourneau V, Blain H, et al. Osteoporosis and ischemic cardiovascular disease[J]. Joint Bone Spine, 2017, 84(4):427-432.

5 Lello S, Capozzi A, Scambia G. Osteoporosis and cardiovascular disease: an update[J]. Gynecol Endocrinol, 2015, 31(8):590-4.

${ }^{6}$ Zeng Y, Wu J, He X, et al. Mechanical microenvironment regulation of age-related diseases involving degeneration of human skeletal and cardiovascular systems[J]. Prog Biophys Mol Biol, 2019,148:54-59.

7 New SE, Aikawa E. Cardiovascular calcification: an inflammatory disease[J]. Circ J, 2011, 75(6):1305-13.

8 García-Gómez MC, Vilahur G. Osteoporosis and vascular calcification: A shared scenario[J]. Clin Investig Arterioscler, 2020,32(1):33-42.

9 Durham AL, Speer MY, Scatena M, et al. Role of smooth muscle cells in vascular calcification: Implications in atherosclerosis and arterial stiffness[J]. Cardiovasc Res, 2018, 114(4):590-600.

10 Albanese I, Khan K, Barratt B, et al. Atherosclerotic Calcification: Wnt Is the Hint[J]. J Am Heart Assoc, 2018, 7(4).

11 Vassalle C, Mazzone A. Bone loss and vascular calcification: A bi-directional interplay? [J]. Vascul Pharmacol, 2016, 86:77-86.

12 Patel JJ, Bourne LE, Davies BK, et al. Differing calcification processes in cultured vascular smooth muscle cells and osteoblasts[J]. Exp Cell Res, 2019,380(1):100-113.

13 Wen L, Chen J, Duan L, et al. Vitamin K-dependent proteins involved in bone and cardiovascular health (Review) [J]. Mol Med Rep, 2018,18(1):3-15.

${ }_{14}$ Millar SA, Patel H, Anderson SI, et al. Osteocalcin, Vascular Calcification, and Atherosclerosis: A Systematic Review and Meta-analysis[J]. Front Endocrinol (Lausanne), 2017,8:183.

15 Tacey A, Qaradakhi T, Brennan-Speranza T, et al. Potential Role for Osteocalcin in the Development of Atherosclerosis and Blood Vessel Disease[J]. Nutrients, 2018, 10(10).

16 Rashdan NA, Sim AM, Cui L, et al. Osteocalcin Regulates Arterial Calcification Via Altered Wnt Signaling and Glucose Metabolism[J]. J Bone Miner Res, 2020, 35(2):357-367.

17 Knez A, Becker C, Becker A, et al. Determination of coronary calcium with multi-slice spiral computed tomography: a comparative study with electron-beam CT[J]. Int J Cardiovasc Imaging, 2002, 18(4):295-303. 
${ }_{18}$ Agatstion AS, Janowitz WR, Hildner FJ, et al. Quantification of coronary artery calcium using ultrafast computed tomography [J]. J Am Coll Cardiol, 1990, 15(4):827-832.

19 Krohn JB, Hutcheson JD, Martínez-Martínez E, et al.Extracellular vesicles in cardiovascular calcification: expanding current paradigms[J]. J Physiol, 2016, 594(11):2895-903.

20 Nakahara T, Dweck MR, Narula N, et al. Coronary Artery Calcification From Mechanism to Molecular Imaging[J]. JACC Cardiovasc Imaging, 2017, 10(5):582-593.

21 Nakahara T, Dweck MR, Narula N, et al. Coronary Artery Calcification: From Mechanism to Molecular Imaging[J]. JACC Cardiovasc Imaging, 2017, 10(5):582-593.

22 Gul'ašová Z, Guerreiro SG, Link R, et al. Tackling endothelium remodeling in cardiovascular disease[J]. J Cell Biochem, 2020, 121(2):938-945.

${ }^{23}$ Leem J, Lee IK. Mechanisms of vascular calcification: The pivotal role of pyruvate dehydrogenase kinase 4[J]. Endocrinol Metab, 2016, 31(1):52-61.

${ }^{24}$ Tesfamariam B. Involvement of Vitamin K-Dependent Proteins in Vascular Calcification[J]. J Cardiovasc Pharmacol Ther,2019,24(4):323-333.

25 Clemente A, Traghella I, Mazzone A, et al. Vascular and valvular calcification biomarkers[J]. Adv Clin Chem, 2020, 95:73-103.

${ }^{26}$ Budoff MJ, Young R, Burke G, et al. Ten-year association of coronary artery calcium with atherosclerotic cardiovascular disease (ASCVD) events: the multi-ethnic study of atherosclerosis (MESA) [J]. Eur Heart J, 2018, 39(25):2401-2408.

27 Yeboah J, McClelland RL, Polonsky TS, et al. Comparison of novel risk markers for improvement in cardiovascular risk assessment in intermediate-risk individuals[J]. JAMA, 2012, 308(8):788-95.

28 Schamroth N, Gutstein A, Kornowski R. Coronary artery Calcium score: Where do We stand? Current uses and implications in asymptomatic patients. Isr Med Assoc J, 2017, 19(4):214-215.

29 Goff DC Jr, Lloyd-Jones DM, Bennett G, et al. 2013 ACC/AHA guideline on the assessment of cardiovascular risk: a report of the American college of cardiology/American heart association task force on practice guidelines[J]. Circulation, 2014,129(25):49-73.

30 Mori H, Torii S, Kutyna M, et al. Coronary Artery Calcification and its Progression: What Does it Really Mean? [J]. JACC Cardiovasc Imaging, 2018, 11(1):127-142.

31 Chen J, Budoff MJ, Reilly MP, et al. Coronary Artery Calcification and Risk of Cardiovascular Disease and Death Among Patients With Chronic Kidney Disease[J]. JAMA Cardiol, 2017, 2(6):635-643.

32 Polonskaya YV, Kashtanova EV, Murashov IS, et al. Associations of Osteocalcin, Osteoprotegerin, and Calcitonin with Inflammation Biomarkers in Atherosclerotic Plaques of Coronary Arteries[J]. Bull Exp Biol Med, 2017, 162(6):726-729.

33 Seidu S, Kunutsor SK, Khunti K. Association of circulating osteocalcin with cardiovascular disease and intermediate cardiovascular phenotypes: systematic review and meta-analysis[J]. Scand Cardiovasc J, 2019, 53(6):286-295.

34 Kim KM, Lim S, Moon JH, et al. Lower uncarboxylated osteocalcin and higher sclerostin levels are significantly associated with coronary artery disease[J]. Bone,2016,83:178-183.

35 Zwakenberg SR, van der Schouw YT, Schalkwijk CG, et al. Bone markers and cardiovascular risk in type 2 diabetes patients[J]. Cardiovasc Diabetol, 2018, 17(1):45.

36 Mathold K, Wanby P, Brudin L, et al. Alterations in bone turnover markers in patients with noncardio-embolic ischemic stroke[J]. PLoS One, 2018, 13(11). 